\title{
Efficacy of generic alendronate and ibandronate in post meno- pausal osteoporosis
}

\author{
N P Somasundaram ${ }^{1}$, N I Thalagala², D M M K Saranapala ${ }^{3}$, M R Sumanetilleke ${ }^{3}$ C N Antonypillai ${ }^{3}$ \\ Sri Lanka Journal of Diabetes Endocrinology and Metabolism 2011; 1: 14-17 \\ (Index words: efficacy, generic, alendronate, osteoporosis)
}

\begin{abstract}
Objective: To determine whether generic non innovator once weekly oral alendronate is as effective as the innovator once oral Ibandronate.

Method: This was an open label, prospective, randomized control trial conducted at the endocrine unit at the National Hospital of Sri Lanka. Postmenopausal women with a BMD T score of <-1.0 were recruited for the trial after excluding those with secondary causes for osteoporosis. Prior to the start of the study all subjects were given a single injection of Vitamin $D_{3} 200000 \mathrm{IU}$. They were thereafter randomized to the alendronate and ibandronate arms. Alendronate arm received alendronate $70 \mathrm{mg}$ once weekly and the ibandronate arm received ibandronate $150 \mathrm{mg}$ once monthly for 3 months. Serum Beta crosslaps (CTX) was measured before and after bisphosphonate treatment. The treatment outcome was assessed using two parameters; i) percentage reduction of CTX and ii) treatment success, defined as greater than $35 \%$ reduction in baseline CTX.
\end{abstract}

Results: Out of 77 women who entered the study 32 women in the alendronate group and 39 women in the ibandronate group completed treatment with 3 drop outs in each group. Mean ages, age since menopause and pre treatment CTX were similar in both groups.

Percentage reduction in beta cross laps levels showed a marginally significant difference favouring the alendronate group $(\mathrm{P}<0.05)$.

Treatment success rates were 93.8 percent in the alendronate group and 89.7 percent in the ibandronate group. The difference was not statistically significant.

Conclusions: Our study shows that the generic preparation of alendronate is effective in the treatment of osteoporosis.

\section{Introduction}

Most patients with post menopausal osteoporosis (PMO) live in developing countries and the proportion is expected to reach $75 \%$ by the year 2050 (1). Cost of drugs to the patients is an important factor to be considered when managing patients in developing countries.

Bisphosphonates are the mainstay of treatment of PMO with proven efficacy in fracture reduction, safety and tolerability (2-6). Alendronate and ibandronate are the commonly used oral bisphosphonates. Comparison of these two drugs has shown that one is not inferior to the other (7). Unfortunately the innovator preparations of these drugs are expensive and beyond the reach of the patients in developing countries. There are generic preparations of alendronate for a reasonable price where they should be almost as effective as the original product in theory. Unfortunately there is insufficient scientific data on bioavailability and effect of these drugs as most landmark studies have used the expensive innovator preparations. Furthermore this concern is justified as only less than $1 \%$ of the oral bisphosphanates is absorbed (8).

Inhibition of bone resorption by bisphosphonates can be used as a surrogate marker of their efficacy. Beta crosslaps (CTX) the $\mathrm{C}$ terminal telopeptide of the triple helix arrangement assumed by type 1 collagen in the bone matrix is one such marker. CTX shows a high sensitivity and specificity for monitoring individual response to antiresorptive therapy (9). The serum CTX assay shows greater utility for assessing efficacy of antiresorptive treatment than some previously described markers such as urinary NTX and DPD (10). More than 35 percent decrease in CTX from the baseline has been used as an indicator of therapeutic success (11). The level of reduction too correlated reasonably well with BMD at 2 years (12).

${ }^{1}$ Consultant Endocrinologist, ${ }^{3}$ Senior Registrar, Diabetes and Endocrine Unit, National Hospital of Sri Lanka, Colombo, ${ }^{2}$ Public Health Specialist, Child Health Unit - 2, Family Health Bureau, Sri Lanka. 
The objective of our study was to determine whether generic alendronate is as effective as the innovator oral Ibandronate; we did this by comparing the efficacy of non innovator once weekly oral generic alendronate with the once monthly innovator oral ibandronate using serum beta cross laps.

\section{Methodology}

\section{Study design}

This was an open label, prospective, randomized control trial.

\section{Setting}

The endocrine unit at the National Hospital of Sri Lanka.

\section{Study participants}

Postmenopausal women with a BMD T score of $<-1.0$ measured by a central DXA scanner (Hologic Discovery W, QDR system software version 12.4.) were recruited for the trial after obtaining their informed written consent. Those who had taken a bisphosphonate or hormone replacement therapy irrespective of the dose or duration of treatment and those who had secondary causes for osteoporosis were excluded from the study. Secondary causes were screened for using an interviewer administered questionnaire, physical examination and biochemical tests including complete blood count, erythrocyte sedimentation rate, serum calcium (ionized or total), serum phosphate, alkaline phosphotase, serum creatinine and thyroid stimulating hormone concentration.

\section{Method}

Prior to the start of the study all subjects were given a single injection of Vitamin $\mathrm{D}_{3} 200000$ IU to treat any possible vitamin D deficiency or insufficiency as vitamin $\mathrm{D}$ was not measured. They were thereafter randomized to the alendronate and ibandronate arms using computer generated random numbers. One week after the vitamin D injection before the start of bisphosphonates fasting CTX level was measured using (Elesys Beta crosslaps, Roche Germany, Elesys 1010). The alendronate group was given oral generical endronate (Osteofos ${ }^{\circledR}$, Cipla, India) $70 \mathrm{mg}$ weekly. The other group received innovator ibandronate (Bonviva ${ }^{\circledR}$, Roche, Switzerland) $150 \mathrm{mg}$ monthly.

The subjects were reviewed monthly and one month's supply of drugs prescribed. Compliance was checked by observing the blister pack for any remaining tablets. Both groups also received Calcitriol $0.25 \mathrm{mcg}$ daily and elemental calcium $1500 \mathrm{mg}$ per day irrespective of the dietary intake.
At the end of three months of therapy the CTX was re-measured. The treatment outcome was assessed using two parameters; i) percentage reduction of CTX and ii) treatment success, defined as greater than $35 \%$ reduction in baseline CTX (11).

\section{Statistical analysis}

The per-protocol population was analysed. The percentage reduction of CTX values between the two groups was compared using Mann Whitney U test. The difference in treatment success between the two groups was compared using Fisher's Exact test.

Finally the post treatment CTX between the two groups was compared using ANCOVA test. As the pre treatment BMD was significantly different between the two groups it was taken as a covariate in the ANCOVA analysis. The data were analysed using SPSS 17.

\section{Results}

77 women who fulfilled the inclusion and exclusion criteria were recruited for the study.

\section{Profiles of the samples}

There were 32 women in the alendronate group and 39 women in the ibandronate group who completed treatment with 3 drop outs in each group. Mean ages were comparable with alendronate group 60.6 years (SD 5.95) years and ibandronate group 62.1 years (SD 7.05) $(\mathrm{t}=$ $-0.308, d f=69 p=0.195)$. There was no statistically significant difference in the age since menopause in the alendronate group (12.3 years (SD 6.61)] and ibandronate group [15.41 years $(\mathrm{SD} 8.19)](\mathrm{t}=-1.725, \mathrm{df}=67 \mathrm{p}=0.089)$. The pretreatment BMD was $0.781 \mathrm{~g} / \mathrm{cm}^{2}$ (SD 0.14) in the alendronate group and $0.694 \mathrm{~g} / \mathrm{cm}^{2}$ (SD 0.11) in the ibandronate group showing a significant difference $(t=2.845, d f=67 p<0.01)$. The levels of pretreatment CTX did not show any significant difference between the two groups. (alendronate $0.706 \mathrm{ng} / \mathrm{ml}, \mathrm{SD}=0.35$, ibandronate $0.825 \mathrm{ng} / \mathrm{ml}, \mathrm{SD}=0.35,(\mathrm{t}=-1.429, \mathrm{df}=69 \mathrm{p}=0.157)$.

\section{Percentage reduction of $C T X$}

The Mann Whitney $U$ test values for percentage reduction in beta cross laps levels showed a marginally significant difference favouring the alendronate group. $(\mathrm{U}=448, \mathrm{Z}=-2.03, \mathrm{~W}=1228, \mathrm{P}=0.042)$. The mean percentage reduction of CTX was $41.5 \%$ for alendronate and $31.49 \%$ for ibandronate. The median percentage reduction of CTX was $67 \%$ for alendronate and $61 \%$ for ibandronate.

Analysis of Co Variance (ANCOVA) was used to compare the two groups in terms of post treatment CTX level. ANCOVA was conducted using Univariate Generalised Linear Model. As the base line analysis showed that the BMD value of ibandronate group $\left(0.694 \mathrm{~g} / \mathrm{cm}^{2}\right)$ 
was significantly lower than the alendronate group $(0.781$ $\mathrm{g} / \mathrm{cm}$ ) the pretreatment CTX was adjusted in the ANOVA by considering the pre treatment BMD as a covariate. After the adjustment there was no significance in the reduction of CTX level $(\mathrm{P}=0.285)$.

\section{Treatment success}

The success rates were 93.8 percent $(n=30 / 32)$ in the alendronate group and 89.7 percent $(n=35 / 39)$ in the ibandronate group. The difference was not statistically significant. (Fisher's Exact test $\mathrm{p}=0.683$ )

\section{Discussion}

Our findings show that oral generic alendronate once a week therapy, available at an affordable price is as effective as oral innovator ibandronate once a month therapy in terms of reduction of CTX at 3 months.

The MOTION study which compared once a month oral ibandronate against once a week oral alendronate showed that both drugs were comparable in efficacy (7). The main difference of our study from the MOTION study is that a generic alendronate was used instead of the branded product used in the latter (7).

The reduction of CTX by each drug had been similar to previous trials which did not have a head to head comparison. Reginster JY et al used CTX to compare the efficacy of two doses of ibandronate (100 mg vs $150 \mathrm{mg}$ ). Here ibandronate $150 \mathrm{mg}$ showed a reduction on in CTX as $56.7 \%$ which is quite similar to the reduction shown in our study (61\%). (13) $47.2 \%$ to $84.7 \%$ reduction of CTX had been noted in trials using Alendronate which is again comparable to our values $(70 \%)(14,15)$.

According to MOTION study the reduction in CTX was greater with alendronate at 3 months but at 6 months both drugs had a similar effect (7). Our study was concluded at 3 months. But this does not deny the fact that generic alendronate was non-inferior to ibandronate in our study.

\section{Significance of the study}

The weakest link in the treatment of osteoporosis is compliance (16) and high cost will add to this effect especially in developing countries where patients often cannot afford expensive drugs. This study proves that the generic preparation of alendronate is equally effective as the innovator ibandronate in reduction of bone resorption markers. This study also provides supportive evidence to physicians in the third world who mainly prescribe the generic products of alendronate which is more affordable.

\section{Limitations}

This was a 3 month study and used only a surrogate marker of treatment efficacy i.e. CTX. BMD has more evidence as a marker for treatment efficacy than CTX although early changes in CTX has been shown to predict long term effects on BMD (17). The ideal measure would have been long term follow up of these patients for fracture reduction.

Technically this was an open label study but the CTX measurements were done by a lab where the staff had no role in the research and they were blind to the two groups in the study.

\section{Conclusion}

In conclusion our study shows that the generic preparation of alendronate is effective in the treatment of osteoporosis.

\section{References}

1. Cooper C, Campion G, Melton LJ, 3rd. Hip fractures in the elderly: a world-wide projection. Osteoporos Int 1992; 2(6): 285-9.

2. Cummings SR, Black DM, Thompson DE, Applegate WB, Barrett-Connor E, Musliner TA, et al. Effect of alendronate on risk of fracture in women with low bone density but without vertebral fractures: results from the Fracture Intervention Trial. JAMA 1998; 280(24): 2077-82.

3. Ensrud KE, Black DM, Palermo L, Bauer DC, BarrettConnor E, Quandt SA, et al. Treatment with alendronate prevents fractures in women at highest risk: results from the Fracture Intervention Trial. Arch Intern Med 1997; 157(22): 2617-24.

4. Felsenberg D, Miller P, Armbrecht G, Wilson K, Schimmer RC, Papapoulos SE. Oral ibandronate significantly reduces the risk of vertebral fractures of greater severity after 1, 2, and 3 years in postmenopausal women with osteoporosis. Bone 2005; 37(5): 651-4.

5. Ho AY, Kung AW. Efficacy and tolerability of alendronate once weekly in Asian postmenopausal osteoporotic women. Ann Pharmacother 2005; 39(9): 1428-33.

6. Levis S, Quandt SA, Thompson D, Scott J, Schneider DL, Ross PD, et al. Alendronate reduces the risk of multiple symptomatic fractures: results from the fracture intervention trial. J Am Geriatr Soc 2002; 50(3): 409-15.

7. Miller PD, Epstein S, Sedarati F, Reginster JY. Oncemonthly oral ibandronate compared with weekly oral alendronate in postmenopausal osteoporosis: results from the head-to-head MOTION study. Curr Med Res Opin 2008; 24(1): 207-13.

8. Lin JH. Bisphosphonates: a review of their pharmacokinetic properties. Bone 1996; 18(2): 75-85.

9. Christgau S, Bitsch-Jensen O, Hanover Bjarnason N, Gamwell Henriksen E, Qvist P, Alexandersen P, et al. Serum CrossLaps for monitoring the response in individuals undergoing antiresorptive therapy. Bone 2000; 26(5): 505-11. 
10. Reiko O, Kiyoshi N, Masaaki I, Takami M, Hiroshi N, Hideki M, et al. Clinical Evaluation of the Elecsys BCrossLaps Serum Assay, a New Assay for Degradation Products of Type I Collagen C-Telopeptides. Clinical Chemistry 2001; 47: 1410-4.

11. Delmas PD, Eastell R, Garnero P, Seibel MJ, Stepan J. The use of biochemical markers of bone turnover in osteoporosis. Committee of Scientific Advisors of the International Osteoporosis Foundation. Osteoporos Int 2000; 11 (Suppl 6): S2-17.

12. Leeming D, Alexandersen P, Karsdal M, Qvist P, Schaller S, Tankó L. An update on biomarkers of bone turnover and their utility in biomedical research and clinical practice. European Journal of Clinical Pharmacology 2006; 62(10): 781-92.

13. Reginster J, Wilson K, Dumont E, Bonvoisin B, Barrett J. Monthly oral ibandronate is well tolerated and efficacious in postmenopausal women: results from the monthly oral pilot study. Journal of Clinical Endocrinology and Metabolism 2005; 90(9): 5018-24.

14. Chailurkit L, Jongjaroenprasert W, Rungbunnapun S, Ongphiphadhanakul B, Sae-tung S, Rajatanavin R. Effect of alendronate on bone mineral density and bone turnover in Thai postmenopausal osteoporosis. Journal Bone Mineral Metabolism 2003; 21(6): 421-7.

15. Choi HJ, Im JA, Kim SH. Changes in bone markers after once-weekly low-dose alendronate in postmenopausal women with moderate bone loss. Maturitas 2008; 60(2): 170-6.

16. Compston J, Seeman E. Compliance with osteoporosis therapy is the weakest link. Lancet 2006; 368(9540): 973-4.

17. Greenspan SL, Rosen HN, Parker RA. Early changes in serum $\mathrm{N}$-telopeptide and C-telopeptide cross-linked collagen type 1 predict long-term response to alendronate therapy in elderly women. J Clin Endocrinol Metab 2000; 85(10): 3537-40. 